\title{
Home Environment and Noise Annoyance in a National Sample of Multi-Family Buildings in Sweden- Associations with Stress-Related Symptoms
}

Juan Wang ( $\boldsymbol{\sim}$ juan.wang@medsci.uu.se)

Department of Medical Sciences, Occupational and Environmental Medicine, Uppsala University, SE-751 85 Uppsala, Sweden

Dan Norbäck

Department of Medical Sciences, Occupational and Environmental Medicine, Uppsala University, SE-751 85 Uppsala, Sweden

\section{Research Article}

Keywords: noise annoyance, stress-related symptoms, building factors, renting, urbanization, traffic noise

Posted Date: August 26th, 2021

DOI: https://doi.org/10.21203/rs.3.rs-712568/v1

License: (9) (7) This work is licensed under a Creative Commons Attribution 4.0 International License. Read Full License 


\section{Abstract}

Background: Poor acoustic condition at home can have negative health impact. The aim was to investigate home environment factors and stress symptoms associated with noise annoyance.

Methods: All adults ( $\geq 18 \mathrm{y}$ ) registered in selected apartments in Sweden were invited to participate in a questionnaire survey including medical questions and personal factors. Totally 5775 adults participated (response rate $46 \%$ ). Information on home environment was obtained through an indoor environment questionnaire. Two-level logistic regression models (individual, municipality) were performed to estimate associations.

Results: Totally $11.2 \%$ reported any frequent noise at home. Voice/radio/TV/music/similar sounds from neighbours (13.2\%) and scraping sound/footsteps/thumping from neighbours (16.5\%) and road traffic (16.1\%). Younger participants reported more noise annoyance and more stress-related symptoms. Females were more sensitive to noise from neighbours and traffic noise causing severe effects. Noise annoyance was related to tiredness, headache and difficulty concentrating ( $O R=1.70-8.19)$, and noise annoyance from many sources was related to a higher risk of symptoms. A warmer climate $(O R=1.30)$, buildings constructed from 1961-1985 (OR=1.50-1.85), renting (OR=1.83) and living alone (OR=1.42) were related to more noise annoyance. A warmer climate $(\mathrm{OR}=1.95)$, higher municipality population density $(\mathrm{OR}=1.24)$, a longer living time $(\mathrm{OR}=1.34)$, construction year $(1961-1975)(\mathrm{OR}=2.42)$, renting $(\mathrm{OR}=1.80-2.32)$, living above ground floor $(\mathrm{OR}=1.45)$ and having a bathroom fan $(\mathrm{OR}=1.84)$ were associated with increased noise annoyance from neighbours. Factors associated with increased noise annoyance from installations or ventilation/fans/heat pumps included a warmer climate, higher municipality population density, construction year (1961-1995), renting and any mechanical ventilation. Higher municipality population density, construction year (especially 1961-1985) and renting were associated with more noise annoyance from traffic (OR=1.77-3.92). Renting (OR=1.73) and living above ground floor $(\mathrm{OR}=1.60)$ were related to more severe traffic noise effects.

Conclusions: The present study shows that noise annoyance may cause stress-related symptoms. Younger age, female gender, living alone, a longer living time, a warmer climate, higher municipality population density and building factors (renting, construction period 1961-1975, living above ground floor and mechanical ventilation system) were related to more noise annoyance.

\section{Background}

Noise pollution, along with air pollution and water pollution, are considered to be three major forms of environmental pollution (1). Environmental noise pollution corresponds to noise caused by road, rail and airport traffic, industry, construction, as well as some other outdoor activities. Noise can cause both auditory and non-auditory health effects (2). Auditory health effects include tinnitus and noise induced hearing loss $(2,3)$. Non-auditory health effects can include sleep disturbances, mental disturbances (stress, mood changes), physical effects (fatigability, headaches), cognitive and learning disorders, cardiovascular effects, etc (2). The mechanisms of the non-auditory noise-induced cardiovascular and metabolic consequences were discussed in one recent review (4)

There are international guidelines aiming to protect humans from harmful exposure to environmental noise. The World Health Organization (WHO) Guidelines for Community Noise 1999 recommend less than 30 A-weighted decibels (dBA) in bedrooms during the night for good sleep quality and less than 35 dBA in classrooms to allow good teaching and learning conditions (5). Levels of $55 \mathrm{dBA}$ is the current WHO guideline for acceptable outdoor noise levels at the most exposed façade of a building (5). It is estimated that in Europe, 125 million people are exposed to road traffic noise greater than 55 decibels (dBA) $L_{\text {den }}$, eight million are exposed to rail traffic noise and three million to aircraft noise above 55 decibels $(d B A) L_{\text {den }}\left(L_{\text {den }}\right.$ is the common EU indicator that corresponds to the average noise level throughout the day, evening and night, to which a citizen is exposed over the period of a year) (6). Almost two million people in Sweden are exposed to average noise levels exceeding national guideline value for outdoor noise (55 dBA) (7).

Noise annoyance, a commonly used marker of noise exposure, can be described as stress reaction involving individual physiological, emotional, cognitive, and behavioural responses $(5,8)$. Noise annoyance could be considered as an early warning signal for other more severe health risks. Standard questions on the degree of noise annoyance have been developed by the International Commission on the Biological Effects of Noise and the International Organization for Standardization, using an 11-point numerical scale and a 5-point semantic scale $(9,10)$. Noise annoyance is regarded as the most important psychological impact of traffic noise exposure (8). Activities such as communication, relaxation/resting/sleeping as well as reading and intellectual work can be especially sensitive to noise disturbances (11). Previous studies suggested that about one-third of annoyance is determined by acoustical factors, another third by nonacoustical factors, while the last third has not been defined yet (12-14). Non-acoustical variables, including person-related factors (e.g., age, education, personal stress and noise sensitivity), social factors (e.g., general evaluation of a noise source, history of noise exposure and quality of life), house-related factors (e.g., windows of living room and/or bedroom oriented toward street, access to a quiet side of the house/apartment, occurrence of sound insulation of the dwelling for windows, front-doors and walls, level of the apartment in a building, own or rented dwelling) and neighbourhood-related factors (distance to the noise source and visibility of the noise source) can play an important role in explaining long-term annoyance (12-17).

Recent reviews indicated comprehensive evidence on road traffic noise exposure and increased risk of ischemic heart diseases and hypertension (18-20). Some evidence was shown on aircraft noise exposure and incidence of hypertension among men (21). One recent review suggested that transportation noise (aircraft, road and rail noise) affects both objectively measured sleep physiology and subjectively assessed sleep disturbance in adults (22). Another recent review concluded that exposure to aircraft noise by objective evaluation was associated with sleep disturbances among adults (23). The WHO report "Burden of disease from environmental noise, 2011" describes that sleep disturbance and annoyance comprise the main burden of environmental noise: one in five individuals has disturbed sleep at night and one in three individuals is annoyed during the daytime because of traffic noise (1). Annoyance was estimated to be the second major health effect of environmental noise after sleep disturbance, with more than 650 thousand healthy life years lost every year (1). 
Research to date has tended to focus on outdoor environmental noise (i.e. road traffic and aircraft noise), while little attention has been paid to indoor noise. The predominant source of noise annoyance in residential quarters is traffic followed by neighbourhood noise. Neighbourhood noises usually include noise of footsteps, sounds with high information content such as language or music, etc. It is in the nature of humans to have their attention drawn to such informative sounds, even at relatively low level. The annoyance potential of neighbourhood noise is therefore relatively high. Most published studies focus on traffic noise caused health problems. Few studies exist on effects of neighbourhood noise on annoyance or health $(24,25)$. One study from Sweden reported that the residents were twice more often annoyed from noise from installation of ventilation and air-conditioning systems than noise from traffic (25). One large European study found neighbourhood noise increased health risk for the cardiovascular system and increased risk of annoyance by depression and migraine among adults (24).

More studies are needed to investigate how annoyance of noise can affect occupants' health. Our study is part of the Building Energy, Technical Status and Indoor Environment (BETSI) study which consists of a stratified random sample of all multi-family buildings in Sweden. The first aim of our study was to estimate the prevalence of noise annoyance (traffic noise and neighbourhood noise) and stress-related symptoms. The second aim was to study associations between noise annoyance and stress-related symptoms. The third aim was to study home environment factors in relation to noise annoyance.

\section{Methods}

\subsection{BETSI study}

The Swedish National Board of Housing, Building and Planning commissioned the BETSI (buildings, energy use, technical status and indoor environment) study in 2006, aiming to obtain representative information of the status of Swedish buildings, as well as indoor environment in relation to occupants' health. The selection of buildings was conducted by Statistics Sweden (SCB) by using a multi-stage sampling procedure (26). Totally 30 Swedish municipalities were selected from a total of 290 municipalities across Sweden through a stratified random selection procedure, taking into account geographic and demographic characteristics (temperature zone and region), and degree of urbanization. Data on all buildings and their construction year from these 30 municipalities were obtained from the central building register in Sweden. Two samples of residential buildings were created: single-family houses and multi-family buildings. Stratified random sampling was applied to sample buildings based on the construction year in five classes (before 1960, 1960-1975, 1976-1985, 1986-1995 and 1996-2005) aiming to get the same number of buildings in each age class. This leads to an over-sampling of new buildings, since most buildings in Sweden are old.

\subsection{Study population and questionnaires}

The present study consisted a subsample of 690 multi-family buildings (including 8841 apartments). The number of adults ( $\geq 18$ years old) living in each apartment was identified by SCB from the Swedish civil registration register. All adults ( $\geq 18$ years) registered in the selected apartments received a personal questionnaire including medical questions and personal factors. Moreover, one indoor environment questionnaire was sent to each apartment. The questionnaires were developed at Department of Medical Science, Uppsala University, based on previous studies (27-32). The postal questionnaire was administered by SCB in the spring of 2008. Two reminders were sent to those who did not reply the first time. Totally 5775 adults participated in the current study and returned the personal questionnaire (46\%), and a total of 4369 indoor environment questionnaires (49\%) were returned.

\subsection{Questions on stress-related symptoms}

One question included in personal questionnaire asked: In the last three months, have you had any of the following symptoms? This question was followed by a list of symptoms. The current study included three stress-related symptoms: (1) tiredness; (2) headache and (3) difficulty concentrating. There were three alternatives for each symptom: yes, often (every week); yes, sometimes; no, never. Those questions originated from the MM-questionnaire from Örebro University Hospital in Sweden (31). Moreover, gender and age were asked in the personal questionnaire.

\subsection{Questions on noise annoyance}

In the personal questionnaire, there were five questions about noise annoyance at home:

(1) Have you been disturbed by noise at home in the last three months? (yes, often (every week); yes, sometimes; no, never);

(2) How much have you been disturbed by sound/noise in the last three months from inside of the building, from: a) lines and pipes; $b$ ) ventilation/fans inside; c) voice/radio/TV/music/similar sounds from neighbours; d) scraping sound/footsteps/thumping/similar sounds from neighbours; e) amusement centre in the property; f) stairwell or elevators. There were sex alternatives for each sub-question: not at all being disturbed; being disturbed not very much; being disturbed a little bit; being disturbed much; being disturbed very much; not existed.

(3) How much have you been disturbed by sound/noise in the last three months from outside of the building, from: a) ventilation/fans/heat pumps; b) road traffic; c) train traffic; d) flight traffic. There were sex alternatives for each sub-question: not at all being disturbed; being disturbed not very much; being disturbed a little bit; being disturbed much; being disturbed very much; not existed.

(4) Does the traffic noise (road, train or flight traffic) cause some of the following effects (severe traffic noise effects)?: a) difficult to hear radio/TV; b) telephone calls being affected; c) normal conversations being affected; $d$ ) rest/relaxation being disturbed; e) difficulties in sleeping; f) being woken up from traffic noise.

A continuous noise score $(0-10)$ was created by adding the number of yes answers to question (2) noise annoyance from sources inside of the building (sex sub-questions) and question (3) noise annoyance from sources outside of the building (four sub-questions). The categorized noise score (0-3) was created: 
coded as 0 if the continuous score equals 0 ; coded as 1 if the continuous score equals 1 ; coded as 2 if the continuous score equals 2 ; coded as 3 if the continuous score equals 3 to 10 .

\subsection{Questions on home environment}

Data on home environment factors were gathered through the questionnaires, including:

(1) Time of been living in the current apartment ( $0-5$ years; more than 5 years);

(2) Total number of teenagers at home;

(3) Total number of children at home;

(4) Total number of persons (children or adults) at home;

(5) Size of the home $\left(\mathrm{m}^{2}\right)$;

(6) Ownership of the current apartment (self-owned; renting);

(7) Location of the apartment (at ground floor/basement; above ground floor);

(8) Any Mechanical ventilation (yes; no);

(9) Bathroom fan (yes; no);

(10) Window opening frequency (everyday; less often).

A variable "crowdedness" was calculated based on total number of persons at home and size of the home (persons/100 $\left.\mathrm{m}^{2}\right)$. Another variable "living alone" was calculated by selecting homes with only one occupant.

Data on building construction year was collected from the National Building Register, Real Property Register and were categorized into five groups: before 1960, 1961-1975, 1976-1985, 1986-1995 and 1996-2005. Data on population and area of each municipality were obtained from wikipedia website (33). Municipality population density (number of persons per $\mathrm{km}^{2}$ ) was calculated based on population and area of each municipality. Data on temperature zone were obtained from a Swedish government report (34). Furthermore, municipality population density was divided to four quartiles: Quartile $1 \leq 25 \%$; $25 \%$ $<$ Quartile $2 \leq 50 \% ; 50 \%<$ Quartile 3 $\leq 75 \% ; 75 \%<$ Quartile $4 \leq 100 \%$. Temperature zone of each municipality was coded as: 1, inner part of north Sweden (Norrland); 2, costal part of Norrland and some inner part of Svealand; 3, Svealand (main parts); 4, Göteland (main parts). A higher number indicates a warmer climate.

\subsection{Statistical analysis}

Chi-square test was applied to compare the prevalence of noise annoyance sources and stress-related symptoms between subgroups (stratified for gender, age, ownership). Initially, factor analysis was performed for two noise factors, including noise from inside of the building (sex variables) and noise from outside of the building (four variables). Then, factor analysis was applied for sex severe traffic noise effects. Two-level logistic regression models (individual, municipality) were performed to estimate associations between noise annoyance sources and stress-related symptoms. Interaction analyses were performed to investigate how gender, age, smoking and ownership affect the associations between noise annoyance sources and respective stress-related symptom. Similar two-level models were conducted to estimate the associations between categorized noise score and stress-related symptoms. Furthermore, similar two-level models were applied to estimate associations between building and home environment factors and different noise annoyance sources. Moreover, mutual adjustment logistic regression model (two-level) was performed including all exposure variables (building and home environment factors) with $\mathrm{p}<0.2$, which were selected from the respective single exposure model.

The statistical analysis was conducted with STATA 15.1 (STATA Corp, Texas, USA) and SPSS 25.0 (SPSS Inc., Chicago, IL, USA). Associations were expressed as odds ratios (OR) with a $95 \%$ confidence interval $(\mathrm{Cl})$ for ordinal regression and logistic regression models. In all statistical analyses, two-tailed tests and a $5 \%$ level of significance were applied, except for the interaction analyses. For the interaction analyses, two-tailed tests and a $10 \%$ level of significance were applied.

\section{Results}

A total of 5775 personal (46\%) and 4369 indoor environment (49\%) questionnaires were returned. Among the returned personal questionnaires, 73 had no information on gender, and 49 had no information on age. The comparison between participants and non-participants was performed by SCB Sweden, and have been published previously (35). All differences between participants and non-participants were small, except for age and civil status. Older persons and married persons had a higher participation rate. Among the participants: $56.5 \%$ were females; $63.5 \%$ aged between 18 to 65 years old, $36.5 \%$ were older than 65 years old; $12.0 \%$ were current smokers.

Prevalence of noise annoyance and stress-related symptoms are shown in Table 1, stratified for gender, age (18-40 y, 40-65 y and over 65 y) and ownership. Totally $11.2 \%$ reported any frequent noise at home in the last three months. Most commonly reported noise from inside of the building were voice/radio/TV/music/similar sounds from neighbours (13.2\%) and scraping sound/footsteps/thumping from neighbours (16.5\%). Road traffic was the most commonly reported noise problem from outdoor sources $(16.1 \%)$. The most common reported severe traffic noise effects were being difficult to hear radio/TV

Page $4 / 21$ 
(3.3\%), rest/relaxation being affected (3.8\%), difficulties in sleeping (3.3\%) and being woken up from traffic noise (3.5\%). Tiredness was the most common symptom among participants (23.1\%). Reporting of headache (8.5\%) and difficulty concentrating (5.5\%) were less common. Females had more symptoms than males (for all symptoms $p<0.001)$. In general, younger participants $(\leq 65 \mathrm{y}$ ) and participants living in rented apartments reported more noise problems as well as more stress-related symptoms.

Associations between noise annoyance and stress-related symptoms are shown in Table 2. All types of noise annoyance were associated with tiredness, headache or difficulty concentrating. Moreover, a higher noise score was related to increased risk of all stress-related symptoms (Table 3 ). 
Table 1

Prevalence of noise annoyance and weekly tiredness, headache and difficulty concentrating.

\begin{tabular}{|c|c|c|c|c|c|c|c|c|c|c|c|c|}
\hline \multicolumn{2}{|l|}{ Environment factors } & $\begin{array}{l}\text { Female } \\
\mathrm{n}= \\
3219^{a} \\
(\%)\end{array}$ & $\begin{array}{l}\text { Male } \\
n= \\
2483 \text { a } \\
(\%)\end{array}$ & $p^{b}$ & $\begin{array}{l}18-40 \\
y n= \\
1433^{c} \\
(\%)\end{array}$ & $\begin{array}{l}41-65 \\
\mathrm{yn}= \\
2204^{\mathrm{c}} \\
(\%)\end{array}$ & $\begin{array}{l}>65 y \\
n= \\
2089^{c} \\
(\%)\end{array}$ & $\mathbf{p}^{b}$ & $\begin{array}{l}\text { Self- } \\
\text { owned } \\
n= \\
2770^{d} \\
(\%)\end{array}$ & $\begin{array}{l}\text { Renting } \\
n= \\
2849 \mathrm{~d} \\
(\%)\end{array}$ & $p^{b}$ & $\begin{array}{l}\text { Total } \\
n= \\
5775 \\
(\%)\end{array}$ \\
\hline Any frequent noise at home & $\begin{array}{l}\text { Often vs. } \\
\text { less or } \\
\text { never }\end{array}$ & 11.3 & 10.7 & 0.501 & 14.8 & 11.7 & 7.9 & $\dot{0} 001$ & 8.1 & 14.2 & $\dot{0} 001$ & 11.2 \\
\hline \multicolumn{13}{|l|}{$\begin{array}{l}\text { Noise from inside of the } \\
\text { building, from }\end{array}$} \\
\hline Lines and pipes & $\begin{array}{l}\text { Much vs. } \\
\text { less/not } \\
\text { at all }\end{array}$ & 8.9 & 8.7 & 0.771 & 12.9 & 9.8 & 4.9 & $\dot{0} 001$ & 5.7 & 12.1 & $\dot{0} 001$ & 8.9 \\
\hline Ventilation/fans inside & $\begin{array}{l}\text { Much vs. } \\
\text { less/not } \\
\text { at all }\end{array}$ & 8.0 & 6.9 & 0.130 & 10.0 & 8.2 & 5.1 & $\dot{0} 001$ & 5.7 & 9.5 & $\dot{0} 001$ & 7.6 \\
\hline $\begin{array}{l}\text { Voice, radio, TV, music or } \\
\text { similar sounds from } \\
\text { neighbors }\end{array}$ & $\begin{array}{l}\text { Much vs. } \\
\text { less/not } \\
\text { at all }\end{array}$ & 14.1 & 12.0 & 0.022 & 21.6 & 13.6 & 6.8 & $\hat{0} .001$ & 8.2 & 18.2 & $\hat{0.001}$ & 13.2 \\
\hline $\begin{array}{l}\text { Scraping sound, footsteps, } \\
\text { thumping or similar sounds } \\
\text { from neighbors }\end{array}$ & $\begin{array}{l}\text { Much vs. } \\
\text { less/not } \\
\text { at all }\end{array}$ & 16.8 & 16.1 & 0.477 & 24.5 & 18.6 & 8.6 & $\hat{0} .001$ & 11.8 & 21.2 & $\hat{0} .001$ & 16.5 \\
\hline $\begin{array}{l}\text { Amusement center in the } \\
\text { property }\end{array}$ & $\begin{array}{l}\text { Much vs. } \\
\text { less/not } \\
\text { at all }\end{array}$ & 2.1 & 2.1 & 0.836 & 3.3 & 2.5 & 0.8 & $\hat{0} .001$ & 1.1 & 3.2 & $\hat{0} .001$ & 2.1 \\
\hline Stairwell, elevators & $\begin{array}{l}\text { Much vs. } \\
\text { less/not } \\
\text { at all }\end{array}$ & 11.0 & 9.6 & 0.098 & 15.0 & 12.3 & 5.0 & $\hat{0} .001$ & 6.6 & 14.5 & $\hat{0.001}$ & 10.5 \\
\hline \multicolumn{13}{|l|}{$\begin{array}{l}\text { Noise from outside of the } \\
\text { building, from }\end{array}$} \\
\hline $\begin{array}{l}\text { Ventilation/fans/warm } \\
\text { pumps }\end{array}$ & $\begin{array}{l}\text { Much vs. } \\
\text { less/not } \\
\text { at all }\end{array}$ & 4.7 & 4.0 & 0.225 & 4.0 & 5.4 & 3.6 & 0.011 & 3.4 & 5.7 & $\dot{0} 001$ & 4.5 \\
\hline Road traffic & $\begin{array}{l}\text { Much vs. } \\
\text { less/not } \\
\text { at all }\end{array}$ & 16.7 & 15.4 & 0.183 & 18.3 & 18.0 & 12.6 & $\dot{0} 001$ & 11.9 & 20.5 & $\hat{0.001}$ & 16.1 \\
\hline Train traffic & $\begin{array}{l}\text { Much vs. } \\
\text { less/not } \\
\text { at all }\end{array}$ & 3.4 & 2.7 & 0.183 & 3.4 & 3.6 & 2.3 & 0.053 & 2.0 & 4.4 & $\dot{0} 001$ & 3.1 \\
\hline Flight traffic & $\begin{array}{l}\text { Much vs. } \\
\text { less/not } \\
\text { at all }\end{array}$ & 3.4 & 3.1 & 0.575 & 3.5 & 4.0 & 2.4 & 0.013 & 2.7 & 4.1 & 0.004 & 3.3 \\
\hline \multicolumn{13}{|l|}{$\begin{array}{l}\text { Severe traffic noise effects } \\
\text { such as }\end{array}$} \\
\hline Difficult to hear radio/TV & $\begin{array}{l}\text { Often vs. } \\
\text { less or } \\
\text { never }\end{array}$ & 3.4 & 3.2 & 0.789 & 4.0 & 3.7 & 2.4 & 0.011 & 2.2 & 4.4 & $\hat{0.001}$ & 3.3 \\
\hline $\begin{array}{l}\text { Telephone calls being } \\
\text { affected }\end{array}$ & $\begin{array}{l}\text { Often vs. } \\
\text { less or } \\
\text { never }\end{array}$ & 1.3 & 1.5 & 0.392 & 1.1 & 1.8 & 1.1 & 0.148 & 1.0 & 1.8 & 0.023 & 1.4 \\
\hline $\begin{array}{l}\text { Normal conversations being } \\
\text { affected }\end{array}$ & $\begin{array}{l}\text { Often vs. } \\
\text { less or } \\
\text { never }\end{array}$ & 1.2 & 1.2 & 0.816 & 1.2 & 1.5 & 0.9 & 0.203 & 0.8 & 1.6 & 0.004 & 1.2 \\
\hline $\begin{array}{l}\text { Rest/relaxation being } \\
\text { affected }\end{array}$ & $\begin{array}{l}\text { Often vs. } \\
\text { less or } \\
\text { never }\end{array}$ & 3.9 & 3.7 & 0.658 & 4.8 & 4.5 & 2.3 & $\dot{0} 001$ & 2.8 & 4.9 & $\dot{0} 001$ & 3.8 \\
\hline \multicolumn{13}{|l|}{ Bold values indicate $p<0.05$} \\
\hline \multicolumn{13}{|c|}{ a participants with missing data on gender $(n=73)$ were excluded. } \\
\hline \multicolumn{13}{|l|}{${ }^{b} \mathrm{p}$ value by $2 \times 3$ Chi-square test. } \\
\hline
\end{tabular}




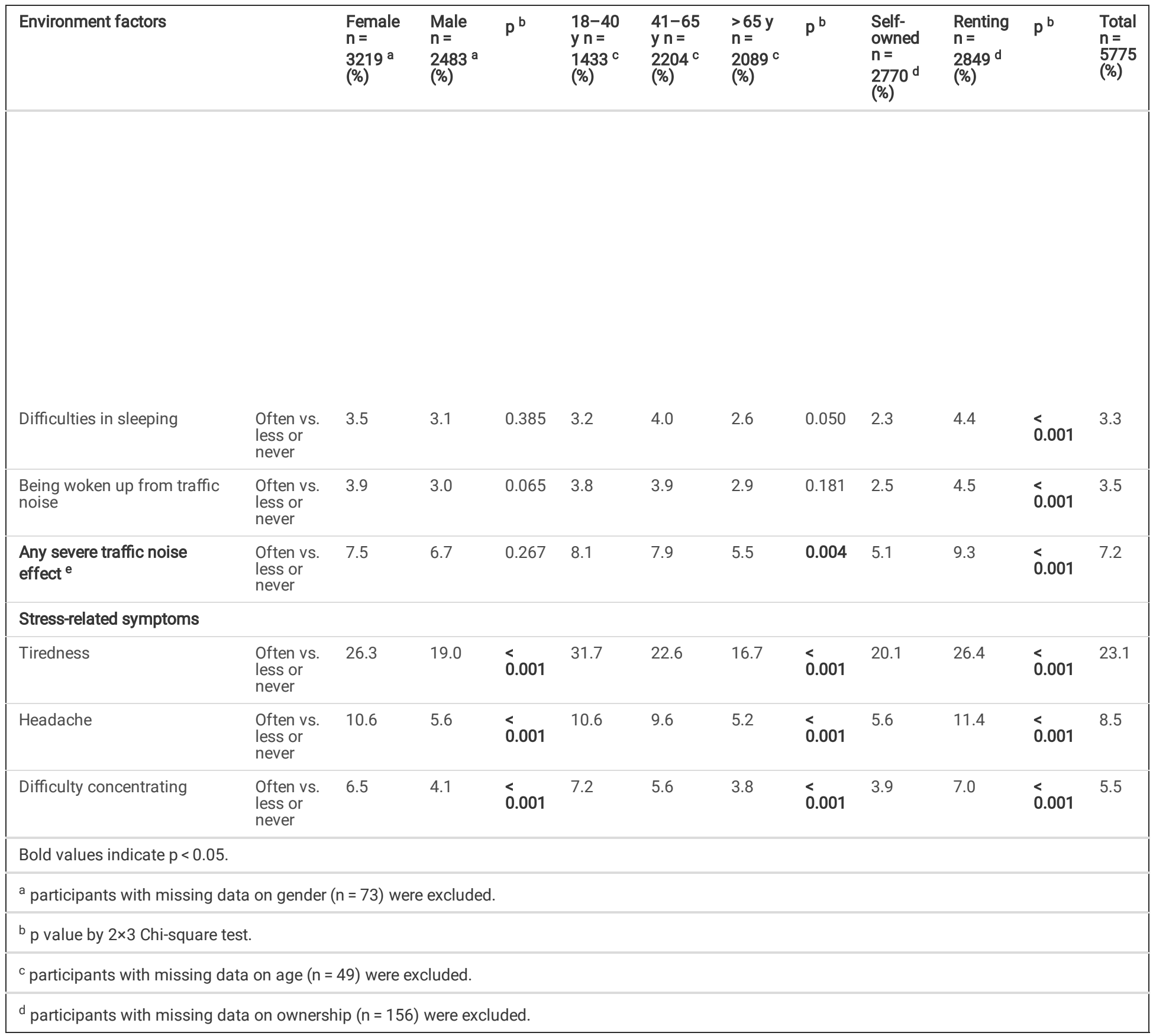

e Any consequence caused by traffic noise was defined as reporting "often" to any of the following traffic noise caused consequences: difficult to hear radio/TV, telephone calls being affected, normal conversations being affected, rest/relaxation being affected, difficulties in sleeping and being woken up from traffic noise. 
Table 2

Associations between noise annoyance and weekly tiredness, headache and difficulty concentrating $\mathrm{OR}(95 \% \mathrm{Cl})^{\mathrm{a}}$.

\begin{tabular}{|c|c|c|c|c|c|c|c|}
\hline Environment factors & & Tiredness & $\mathbf{p}$ & Headache & $\mathbf{p}$ & $\begin{array}{l}\text { Difficulty } \\
\text { concentrating }\end{array}$ & p \\
\hline Any frequent noise at home & $\begin{array}{l}\text { Often vs. less or } \\
\text { never }\end{array}$ & $2.08(1.72,2.51)$ & $\hat{0} 001$ & $3.47(2.71,4.43)$ & $\hat{0.001}$ & $3.68(2.76,4.92)$ & $\dot{0} 001$ \\
\hline \multicolumn{8}{|l|}{ Noise from inside of the building, from } \\
\hline Lines and pipes & $\begin{array}{l}\text { Much vs. } \\
\text { less/not at all }\end{array}$ & $1.84(1.48,2.28)$ & $<.001$ & $2.81(2.13,3.71)$ & $<.001$ & $2.11(1.49,3.00)$ & <. 001 \\
\hline Ventilation/fans inside & $\begin{array}{l}\text { Much vs. } \\
\text { less/not at all }\end{array}$ & $1.85(1.47,2.34)$ & $\dot{0} 001$ & $2.44(1.80,3.32)$ & $<.001$ & $2.49(1.73,3.57)$ & $<.001$ \\
\hline $\begin{array}{l}\text { Voice, radio, TV, music or similar sounds from } \\
\text { neighbors }\end{array}$ & $\begin{array}{l}\text { Much vs. } \\
\text { less/not at all }\end{array}$ & $1.82(1.52,2.18)$ & <. 001 & $2.90(2.28,3.69)$ & $<.001$ & 2.31(1.71,3.13) & $<.001$ \\
\hline $\begin{array}{l}\text { Scraping sound, footsteps, thumping or similar } \\
\text { sounds from neighbors }\end{array}$ & $\begin{array}{l}\text { Much vs. } \\
\text { less/not at all }\end{array}$ & $1.70(1.43,2.01)$ & $\hat{0} .001$ & $2.76(2.19,3.47)$ & $\hat{0} 001$ & $2.23(1.68,2.96)$ & $\dot{0} 001$ \\
\hline Amusement center in the property & $\begin{array}{l}\text { Much vs. } \\
\text { less/not at all }\end{array}$ & $1.42(0.93,2.17)$ & 0.106 & $2.45(1.46,4.09)$ & 0.001 & $2.39(1.30,4.38)$ & 0.005 \\
\hline Stairwell, elevators & $\begin{array}{l}\text { Much vs. } \\
\text { less/not at all }\end{array}$ & $2.07(1.69,2.53)$ & $<.001$ & $3.52(2.73,4.54)$ & $<.001$ & $2.85(2.08,3.92)$ & $\dot{0} 001$ \\
\hline \multicolumn{8}{|l|}{ Noise from outside of the building, from } \\
\hline Ventilation/fans/warm pumps & $\begin{array}{l}\text { Much vs. } \\
\text { less/not at all }\end{array}$ & $1.96(1.47,2.60)$ & $\hat{0} .001$ & $3.73(2.65,5.24)$ & $<.001$ & $3.26(2.17,4.90)$ & <. 001 \\
\hline Road traffic & $\begin{array}{l}\text { Much vs. } \\
\text { less/not at all }\end{array}$ & $1.96(1.67,2.32)$ & $\hat{0} .001$ & $2.44(1.95,3.07)$ & $\begin{array}{l}<.001 \\
0.01\end{array}$ & $2.19(1.65,2.90)$ & <. 001 \\
\hline Train traffic & $\begin{array}{l}\text { Much vs. } \\
\text { less/not at all }\end{array}$ & $1.87(1.33,2.61)$ & $<.001$ & $3.29(2.19,4.94)$ & $\hat{0} .001$ & $2.88(1.78,4.69)$ & $<.001$ \\
\hline Flight traffic & $\begin{array}{l}\text { Much vs. } \\
\text { less/not at all }\end{array}$ & $2.16(1.56,2.97)$ & $<.001$ & $2.70(1.78,4.08)$ & $<.001$ & $2.96(1.84,4.75)$ & $<.001$ \\
\hline \multicolumn{8}{|l|}{ Severe traffic noise effects such as } \\
\hline Difficult to hear radio/TV & $\begin{array}{l}\text { Often vs. less or } \\
\text { never }\end{array}$ & $3.23(2.36,4.41)$ & $<.001$ & $5.57(3.92,7.91)$ & $\begin{array}{l}<.001 \\
0.00\end{array}$ & $5.29(3.55,7.89)$ & <. 001 \\
\hline Telephone calls being affected & $\begin{array}{l}\text { Often vs. less or } \\
\text { never }\end{array}$ & $3.48(2.15,5.62)$ & $<.001$ & $7.10(4.22,11.9)$ & $\begin{array}{l}<.001 \\
0.01\end{array}$ & $6.52(3.67,11.6)$ & <. 001 \\
\hline Normal conversations being affected & $\begin{array}{l}\text { Often vs. less or } \\
\text { never }\end{array}$ & $3.74(2.25,6.22)$ & $\hat{0} .001$ & $5.78(3.33,10.0)$ & $<.001$ & $7.11(3.97,12.7)$ & ¿.001 \\
\hline Rest/relaxation being affected & $\begin{array}{l}\text { Often vs. less or } \\
\text { never }\end{array}$ & $2.91(2.17,3.89)$ & $\dot{0} 001$ & $5.24(3.77,7.28)$ & $\begin{array}{l}< \\
0.001\end{array}$ & $4.13(2.79,6.10)$ & $<.001$ \\
\hline Difficulties in sleeping & $\begin{array}{l}\text { Often vs. less or } \\
\text { never }\end{array}$ & $3.93(2.87,5.38)$ & $\hat{0} .001$ & $8.19(5.81,11.6)$ & $<.001$ & $4.97(3.29,7.50)$ & <. 001 \\
\hline Being woken up from traffic noise & $\begin{array}{l}\text { Often vs. less or } \\
\text { never }\end{array}$ & $3.75(2.77,5.09)$ & $<.001$ & $7.58(5.45,10.5)$ & $<.001$ & $5.79(3.93,8.53)$ & <. 001 \\
\hline Any severe traffic noise effect ${ }^{b}$ & $\begin{array}{l}\text { Often vs. less or } \\
\text { never }\end{array}$ & $2.87(2.30,3.58)$ & $<.001$ & $5.02(3.85,6.55)$ & $\begin{array}{l}< \\
0.001\end{array}$ & $4.74(3.46,6.48)$ & $\begin{array}{l}<.001 \\
0.00\end{array}$ \\
\hline \multicolumn{8}{|l|}{ Bold values indicate $p<0.05$} \\
\hline \multicolumn{8}{|c|}{ a Two-level logistic regression models (individual, municipality). The odds ratios were adjusted for gender, age and smoking. } \\
\hline
\end{tabular}


Table 3

Associations between categorized noise score and weekly tiredness, headache and difficulty concentrating $\mathrm{OR}(95 \% \mathrm{Cl})^{\mathrm{a}}$.

\begin{tabular}{|c|c|c|c|c|c|c|c|c|}
\hline Environment factors & & $\%$ & Tiredness & $p$ & Headache & $p$ & Difficulty concentrating & $\mathbf{p}$ \\
\hline \multirow[t]{4}{*}{ Categorized noise score $^{b}$} & 0 & 62.0 & 1.00 & & 1.00 & & 1.00 & \\
\hline & 1 & 17.7 & $1.64(1.35,1.99)$ & $<0.001$ & $1.85(1.34,2.56)$ & $<0.001$ & $1.41(0.94,2.12)$ & 0.100 \\
\hline & 2 & 8.9 & $1.89(1.48,2.42)$ & $<0.001$ & $2.23(1.52,3.27)$ & $<0.001$ & $1.88(1.18,3.02)$ & 0.008 \\
\hline & 3 & 11.4 & $2.63(2.12,3.26)$ & $<0.001$ & $5.39(4.01,7.24)$ & $<0.001$ & $4.40(3.09,6.25)$ & $<0.001$ \\
\hline
\end{tabular}

Bold values indicate $\mathrm{p}<0.05$

a Two-level logistic regression models (individual, municipality). The odds ratios were adjusted for gender, age and smoking.

${ }^{b}$ A continuous noise score $(0-10)$ was created by adding the number of yes answers to noise from sources inside of the building (sex sub-questions) and noise from sources outside of the building (four sub-questions). The categorized noise score $(0-3)$ was created: coded as 0 if the continuous score equals 0 ; coded as 1 if the continuous score equals 1 ; coded as 2 if the continuous score equals 2; coded as 3 if the continuous score equals 3 to 10 .

Interaction analyses were applied to study if gender, age, smoking and ownership affect the associations between noise annoyance and respective stressrelated symptom. Females were more often tired than men if exposed to traffic noise causing severe effects ( $p$ for interaction 0.013 ). Older subjects got more often headache if exposed to noise at home ( $\mathrm{p}$ for interaction 0.053 with age as continuous variable). Moreover, older subjects got more often headache if exposed to traffic noise causing severe effects ( $p$ for interaction 0.003 with age as continuous variable). Subjects owning their apartment were more often tired if exposed to noise at home, as compared to those renting their apartment ( $p$ for interaction 0.036). Moreover, subjects owning their apartment got more often headache if exposed to traffic noise causing severe effects ( $p$ for interaction 0.054 ). No interaction by smoking was observed on the associations between different types of noise annoyance and headache, tiredness or difficulty concentrating ( $p$ for interaction $>0.1)$.

The initial factor analysis identified three factors: the first factor included voice/radio/TV/music/similar sounds from neighbours, scraping sound/footsteps/thumping/similar sounds from neighbours and noise from stairwell or elevators; the second factor included noise from ventilation/fans inside and noise from ventilation/fans/heat pumps from outside of the building; the third factor included noise from road traffic, train traffic and flight traffic. Noise from lines and pipes and noise from amusement centre in the property were not included in any factor. The second factor analysis for severe traffic noise effects identified two factors: the first factor included difficult to hear radio/TV, telephone calls being affected and normal conversations being affected; the second factor included rest/relaxation being disturbed, difficulties in sleeping and been woken up from traffic noise.

The majority of the participants were living in warmer climate zones (median value for temperature zone is 3 ). The median value of municipality population density is 1179 person $/ \mathrm{km}^{2}$ and the median value of crowdedness is 2.3 person/100 $\mathrm{m}^{2}$. Over half (52.6\%) of the participants have been living in the current apartment more than 5 years. Totally $6.8 \%$ reported any teenager at home and $11.7 \%$ reported any children at home. Less than half ( $40.9 \%$ ) of the participants were living alone. About half (50.7\%) of the participants had rented apartments. The majority of the participants were living above ground floor (81.8\%). Apartments with mechanical ventilation accounted for $45.4 \%$. Only $14.6 \%$ had a bathroom fan. A total of $73.9 \%$ ventilated their homes on a daily basis by opening windows (Table 4).

Construction year 1961-1975, living alone and renting were all associated with any frequent noise at home. Any mechanical ventilation was related to less disturbing noise at home. Three risk factors were identified in the mutual adjustment model including all variables with $p<0.2$ in Table 4: a warmer climate $(\mathrm{OR}=1.30,95 \% \mathrm{Cl} 1.04-1.62 ; \mathrm{p}=0.022)$, construction year $1961-1975(\mathrm{OR}=1.85,95 \% \mathrm{Cl} 1.30-2.61 ; \mathrm{p}=0.001)$ and $1976-1985(\mathrm{OR}=1.57,95 \% \mathrm{Cl} 1.06-2.34$; $p=0.025)$ and renting $(O R=1.83,95 \% \mathrm{Cl} 1.48-2.28 ; p<0.001)$ were all associated with reporting of any frequent noise at home.

Building and home environment factors in relation to noise annoyance from sources inside of the building were analysed (see Table S1 and Table S2 in Additional file 1). Mutual adjustment model including all variables with $p<0.2$ in Table S1 (see Additional file 1) were performed (Table 5). For reporting of noise from lines and pipes, higher municipality population density, construction year 1961-1975 and renting were all risk factors. Construction year 19761985 and 1986-1995, renting and any mechanical ventilation were all associated with more noise from ventilation/fans inside. Daily window opening was related to less noise from ventilation/fans inside. Risk factors for noise from voice/radio/TV/music/similar sounds from neighbours included renting and living above ground floor. Construction year 1986-2005 was associated with less reporting of noise from voice/radio/TV/music/similar sounds from neighbours. Mutual adjustment model including all variables with $\mathrm{p}<0.2$ in Table S2 (see Additional file 1) were applied (Table 6). Living in current apartment more than 5 years and renting were associated with more reporting of scraping sound/footsteps/thumping/similar sounds from neighbours. Living in newer buildings (construction year 1976-2005) was related to less annoyance of such noise. Risk factors for reporting of noise from amusement center in the property included a warmer climate, higher municipality population density, construction year 1961-1975, renting and having a bathroom fan. A warmer climate and renting were associated with reporting of noise from stairwell/elevators. Living in newer buildings (constructed during 1996-2005) was related to less reporting of noise from stairwell or elevators.

Table S3 (see Additional file 1) shows associations between building and home environment factors, noise annoyance from sources outside of the building and any severe traffic noise effect. Mutual adjustment models were applied (Table 7), including all variables with p < 0.2 in Table S3 (see Additional file 1). Four risk factors were identified for noise from ventilation/fans/heat pumps: higher municipality population density, construction year 1961-1995, renting and any mechanical ventilation. Daily window opening was related to less complain of noise from ventilation/fans/heat pumps. Renting was associated with reporting of noise from road traffic. Higher municipality population density, construction year 1961-1985 and 1996-2005 and renting were related to more complain of noise from train traffic. Three factors were associated with reporting of noise from flight traffic: higher municipality population density, 
construction year 1976-1985 and daily window opening. Living in newer buildings (construction year 1996-2005) was related to less complain of noise from flight traffic. Renting and living above ground floor were related to more reporting of any severe traffic noise effect.

Table 4

Associations between environment factors and any frequent noise at home (often vs. less/never) OR(95\% Cl) ${ }^{a}$

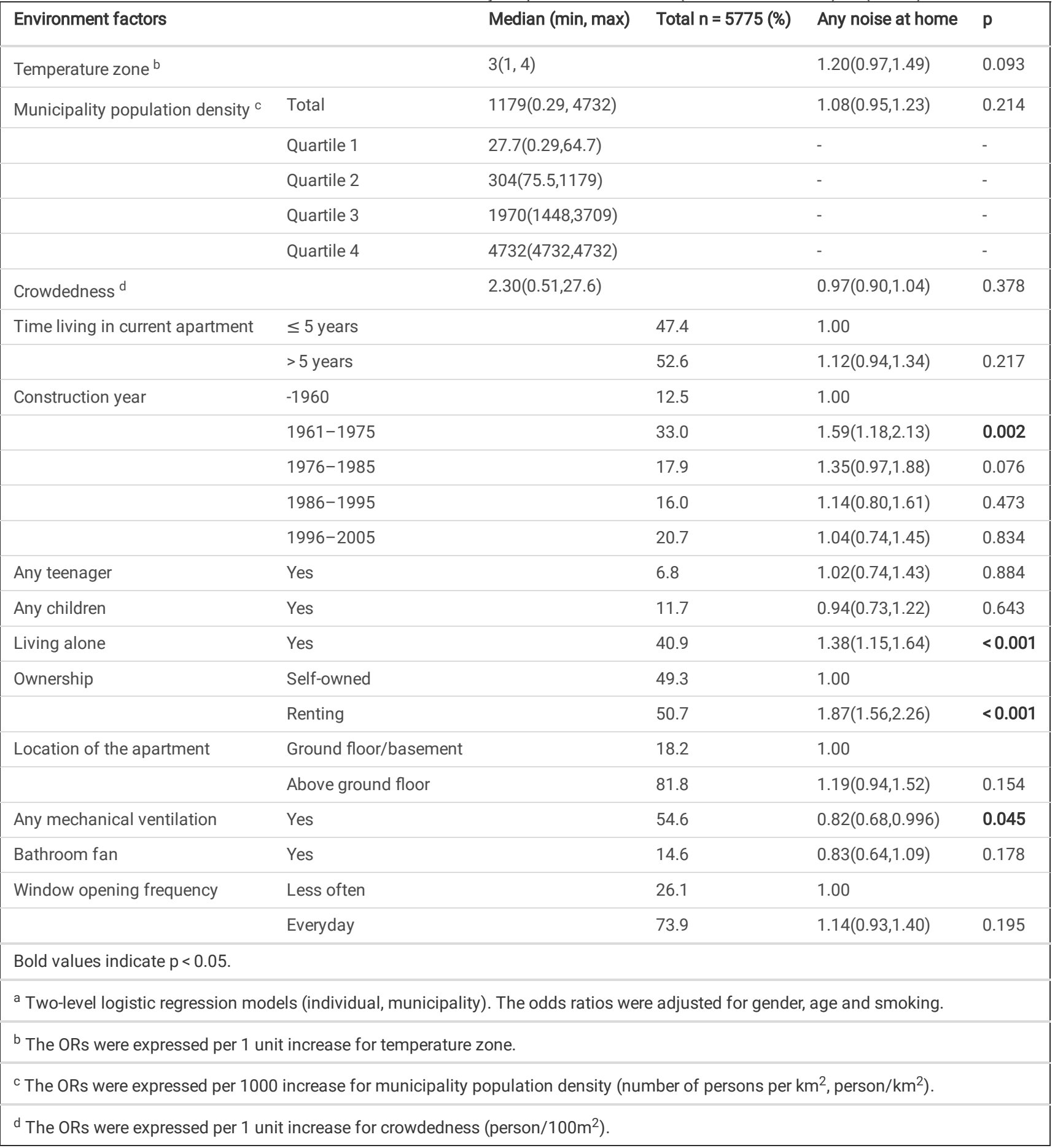


Table 5

Associations between environment factors and noise annoyance from sources inside (much vs. less/not at all) $\mathrm{OR}\left(95 \% \mathrm{Cl}\right.$ ) ${ }^{\text {a }}$.

\begin{tabular}{|c|c|c|c|c|c|c|c|}
\hline Environment factors & & $\begin{array}{l}\text { Lines and } \\
\text { pipes }\end{array}$ & p & $\begin{array}{l}\text { Ventilation/fans } \\
\text { inside }\end{array}$ & $p$ & $\begin{array}{l}\text { Voice, radio, TV, music, similar sounds } \\
\text { from neighbors }\end{array}$ & $p$ \\
\hline $\begin{array}{l}\text { Municipality population } \\
\text { density }{ }^{b}\end{array}$ & & $1.07(1.01,1.14)$ & 0.020 & - & - & - & - \\
\hline \multirow{2}{*}{$\begin{array}{l}\text { Time living in current } \\
\text { apartment }\end{array}$} & $\leq 5$ years & - & - & - & - & 1.00 & \\
\hline & $>5$ years & - & - & - & - & $1.23(0.99,1.52)$ & 0.059 \\
\hline \multirow[t]{5}{*}{ Construction year } & -1960 & 1.00 & & 1.00 & & 1.00 & \\
\hline & $1961-1975$ & $1.86(1.27,2.74)$ & 0.002 & $1.55(0.96,2.52)$ & 0.076 & $1.28(0.96,1.72)$ & 0.094 \\
\hline & 1976-1985 & $1.21(0.77,1.90)$ & 0.403 & $2.40(1.45,3.95)$ & 0.001 & $0.88(0.63,1.24)$ & 0.474 \\
\hline & 1986-1995 & $1.33(0.84,2.11)$ & 0.223 & $2.51(1.51,4.17)$ & $\begin{array}{l}<.001 \\
0.00\end{array}$ & $0.62(0.43,0.91)$ & 0.014 \\
\hline & 1996-2005 & $1.02(0.65,1.61)$ & 0.932 & $0.94(0.54,1.61)$ & 0.813 & $0.34(0.22,0.51)$ & <. 001 \\
\hline \multirow[t]{2}{*}{ Ownership } & Self-owned & 1.00 & & 1.00 & & 1.00 & \\
\hline & Renting & $1.88(1.47,2.39)$ & $\hat{0} .001$ & $1.44(1.12,1.87)$ & 0.005 & $2.07(1.69,2.55)$ & ¿.001 \\
\hline \multirow[t]{2}{*}{$\begin{array}{l}\text { Location of the } \\
\text { apartment }\end{array}$} & $\begin{array}{l}\text { Ground } \\
\text { floor/basement }\end{array}$ & 1.00 & & - & - & 1.00 & \\
\hline & $\begin{array}{l}\text { Above ground } \\
\text { floor }\end{array}$ & $1.09(0.81,1.48)$ & 0.555 & - & - & $1.45(1.10,1.90)$ & 0.008 \\
\hline $\begin{array}{l}\text { Any mechanical } \\
\text { ventilation }\end{array}$ & & $0.82(0.65,1.04)$ & 0.099 & $1.30(1.002,1.69)$ & 0.048 & $1.01(0.82,1.24)$ & 0.953 \\
\hline Bathroom fan & Yes & - & - & - & - & $1.08(0.81,1.43)$ & 0.604 \\
\hline \multirow[t]{2}{*}{$\begin{array}{l}\text { Window opening } \\
\text { frequency }\end{array}$} & Less often & - & - & 1.00 & & - & - \\
\hline & Everyday & - & - & $0.70(0.54,0.90)$ & 0.006 & - & - \\
\hline \multicolumn{8}{|c|}{ Bold values indicate $p<0.05$} \\
\hline \multicolumn{8}{|c|}{$\begin{array}{l}\text { a Two-level logistic regression models (individual, municipality). Mutual adjustment including all variables with } \mathrm{p}<0.2 \text { in Table S1 (see Additional file } 1 \text { ). } \\
\text { The odds ratios were adjusted for gender, age and smoking. }\end{array}$} \\
\hline
\end{tabular}


Table 6

Associations between environment factors and noise annoyance from sources inside (much vs. less/not at all) $\mathrm{OR}(95 \% \mathrm{Cl}){ }^{\text {a }}$

\begin{tabular}{|c|c|c|c|c|c|c|c|}
\hline $\begin{array}{l}\text { Environment } \\
\text { factors }\end{array}$ & & $\begin{array}{l}\text { Scraping sound, footsteps, thumping or } \\
\text { similar sounds from neighbours }\end{array}$ & $p$ & $\begin{array}{l}\text { Amusement center } \\
\text { in the property }\end{array}$ & $p$ & $\begin{array}{l}\text { Stairwell, } \\
\text { elevators }\end{array}$ & $p$ \\
\hline Temperature zone ${ }^{\mathrm{b}}$ & & - & - & $1.95(1.20,3.15)$ & 0.007 & $1.21(1.04,1.41)$ & 0.016 \\
\hline $\begin{array}{l}\text { Municipality } \\
\text { population density } \\
\text { c }\end{array}$ & & - & - & $1.24(1.03,1.49)$ & 0.022 & - & - \\
\hline \multirow{2}{*}{$\begin{array}{l}\text { Time living } \\
\text { in current } \\
\text { apartment }\end{array}$} & $\leq 5$ years & 1.00 & & - & - & 1.00 & \\
\hline & $>5$ years & $1.34(1.10,1.62)$ & 0.003 & - & - & $1.22(0.96,1.54)$ & 0.103 \\
\hline \multirow[t]{5}{*}{ Construction year } & -1960 & 1.00 & & 1.00 & & 1.00 & \\
\hline & $1961-1975$ & $1.23(0.94,1.60)$ & 0.137 & $2.42(1.25,4.67)$ & 0.008 & $1.29(0.93,1.78)$ & 0.132 \\
\hline & $1976-1985$ & $0.67(0.49,0.93)$ & 0.017 & $0.88(0.38,2.03)$ & 0.765 & $0.83(0.56,1.24)$ & 0.365 \\
\hline & 1986-1995 & $0.62(0.44,0.87)$ & 0.006 & $1.32(0.58,3.01)$ & 0.513 & $0.74(0.49,1.13)$ & 0.165 \\
\hline & $1996-2005$ & $0.57(0.41,0.80)$ & 0.001 & $0.66(0.27,1.61)$ & 0.358 & $0.63(0.41,0.96)$ & 0.031 \\
\hline \multirow[t]{2}{*}{ Ownership } & Self-owned & 1.00 & & 1.00 & & 1.00 & \\
\hline & Renting & $1.80(1.50,2.16)$ & $<.001$ & $2.32(1.49,3.63)$ & $\dot{0} 001$ & $2.19(1.73,2.76)$ & $\hat{0} .001$ \\
\hline \multirow[t]{2}{*}{$\begin{array}{l}\text { Location of the } \\
\text { apartment }\end{array}$} & $\begin{array}{l}\text { Ground } \\
\text { floor/basement }\end{array}$ & 1.00 & & - & - & - & - \\
\hline & $\begin{array}{l}\text { Above ground } \\
\text { floor }\end{array}$ & $1.11(0.88,1.40)$ & 0.387 & - & - & - & - \\
\hline $\begin{array}{l}\text { Any mechanical } \\
\text { ventilation }\end{array}$ & & $1.06(0.88,1.28)$ & 0.506 & & & $0.89(0.71,1.11)$ & 0.297 \\
\hline Bathroom fan & Yes & - & - & $1.84(1.16,2.93)$ & 0.010 & - & - \\
\hline \multirow[t]{2}{*}{$\begin{array}{l}\text { Window opening } \\
\text { frequency }\end{array}$} & Less often & - & - & - & - & - & - \\
\hline & Everyday & - & - & - & - & - & - \\
\hline \multicolumn{8}{|c|}{ Bold values indicate $p<0.05$} \\
\hline \multicolumn{8}{|c|}{$\begin{array}{l}\text { a Two-level logistic regression models (individual, municipality). Mutual adjustment including all variables with } p<0.2 \text { in Table S2 (see Additional file } 1 \text { ). } \\
\text { The odds ratios were adjusted for gender, age and smoking. }\end{array}$} \\
\hline \multicolumn{8}{|c|}{ b The ORs were expressed per 1 unit increase for temperature zone. } \\
\hline
\end{tabular}


Associations between environment factors and noise annoyance from sources outside (much vs. less/not at all) $\mathrm{OR}(95 \% \mathrm{Cl})^{\mathrm{a}}$.

\begin{tabular}{|c|c|c|c|c|c|c|c|c|c|c|}
\hline $\begin{array}{l}\text { Environment } \\
\text { factors }\end{array}$ & & $\begin{array}{l}\text { Ventilation/fans/warm } \\
\text { pumps }\end{array}$ & $p$ & Road traffic & $p$ & Train traffic & $p$ & Flight traffic & p & $\begin{array}{l}\text { An } \\
\text { tra } \\
\text { efi } \\
\text { vs } \\
\text { ne }\end{array}$ \\
\hline $\begin{array}{l}\text { Temperature } \\
\text { zone }^{b}\end{array}$ & & $1.22(0.98,1.53)$ & 0.081 & - & - & - & - & - & - & $1 . \AA$ \\
\hline $\begin{array}{l}\text { Municipality } \\
\text { population } \\
\text { density }^{c}\end{array}$ & & $1.13(1.05,1.22)$ & 0.002 & - & - & $1.42(1.02,1.99)$ & 0.039 & $2.00(1.14,3.50)$ & 0.015 & - \\
\hline \multirow{2}{*}{$\begin{array}{l}\text { Time living } \\
\text { in current } \\
\text { apartment }\end{array}$} & $\leq 5$ years & 1.00 & & - & - & - & - & 1.00 & & - \\
\hline & $>5$ years & $0.93(0.67,1.28)$ & 0.641 & - & - & - & - & $1.00(0.71,1.41)$ & 0.989 & - \\
\hline \multirow{5}{*}{$\begin{array}{l}\text { Construction } \\
\text { year }\end{array}$} & -1960 & 1.00 & & 1.00 & & 1.00 & & 1.00 & & 1.C \\
\hline & $1961-1975$ & $1.81(1.01,3.24)$ & 0.046 & $0.92(0.70,1.21)$ & 0.556 & $3.27(1.55,6.92)$ & 0.002 & $1.57(0.91,2.72)$ & 0.106 & 1.C \\
\hline & 1976-1985 & $2.43(1.32,4.47)$ & 0.004 & $0.75(0.54,1.02)$ & 0.067 & $3.19(1.45,7.05)$ & 0.004 & $2.35(1.32,4.17)$ & 0.004 & 1.1 \\
\hline & 1986-1995 & $2.15(1.15,4.03)$ & 0.017 & $0.77(0.56,1.06)$ & 0.110 & $2.18(0.93,5.09)$ & 0.073 & $1.36(0.74,2.49)$ & 0.319 & $0 . \subseteq$ \\
\hline & 1996-2005 & $0.92(0.47,1.80)$ & 0.807 & $0.81(0.59,1.09)$ & 0.167 & $3.92(1.80,8.53)$ & 0.001 & $0.39(0.18,0.84)$ & 0.016 & $0 . \overline{1}$ \\
\hline \multirow[t]{2}{*}{ Ownership } & Self-owned & 1.00 & & 1.00 & & 1.00 & & 1.00 & & 1.C \\
\hline & Renting & $1.55(1.14,2.11)$ & 0.005 & $1.77(1.48,2.13)$ & $<.001$ & $2.52(1.75,3.62)$ & $<.001$ & $1.28(0.91,1.78)$ & 0.152 & 1.7 \\
\hline \multirow{2}{*}{$\begin{array}{l}\text { Location of } \\
\text { the } \\
\text { apartment }\end{array}$} & $\begin{array}{l}\text { Ground } \\
\text { floor/basement }\end{array}$ & - & - & - & - & - & - & - & - & $1 . \mathrm{C}$ \\
\hline & $\begin{array}{l}\text { Above ground } \\
\text { floor }\end{array}$ & - & - & - & - & - & - & - & - & $1 . \epsilon$ \\
\hline $\begin{array}{l}\text { Any } \\
\text { mechanical } \\
\text { ventilation }\end{array}$ & Yes & $1.46(1.06,2.00)$ & 0.020 & $0.95(0.80,1.14)$ & 0.596 & & & & & $0 . c$ \\
\hline $\begin{array}{l}\text { Bathroom } \\
\text { fan }\end{array}$ & Yes & - & - & - & - & - & - & - & - & - \\
\hline \multirow[t]{2}{*}{$\begin{array}{l}\text { Window } \\
\text { opening } \\
\text { frequency }\end{array}$} & Less often & 1.00 & & 1.00 & & - & - & 1.00 & & 1.C \\
\hline & Everyday & $0.64(0.47,0.88)$ & 0.005 & $1.10(0.90,1.33)$ & 0.355 & - & - & $1.56(1.05,2.31)$ & 0.026 & 1.2 \\
\hline
\end{tabular}

Bold values indicate $p<0.05$

a Two-level logistic regression models (individual, municipality). Mutual adjustment including all variables with $p<0.2$ in Table S3 (see Additional file 1 ). The adjusted for gender, age and smoking.

$\mathrm{b}$ The ORs were expressed per 1 unit increase for temperature zone.

${ }^{\mathrm{c}}$ The ORs were expressed per 1000 increase for municipality population density (number of persons per $\mathrm{km}^{2}$, person $/ \mathrm{km}^{2}$ ).

The prevalence of any frequent noise at home and any severe traffic noise effect in different construction periods, stratified by ownership (self-owned/renting) are shown in Fig. 1 and Fig. 4, respectively. The prevalence of severe traffic noise effects was shown in Fig. 3, stratified by ownership. Participants living in buildings constructed during 1961-1975 and 1976-1985 reported more noise problems (Fig. 1 and Fig. 4). Reported any frequent noise at home, severe traffic noise effects and any severe traffic noise effect were all higher among those living in rented apartments as compared to those living in self-owned apartments (Fig. 1, Fig. 3 and Fig. 4). Increased municipality population density (indicated by four quartiles) was related to a higher prevalence of any frequent noise at home (Fig. 2) and a higher prevalence of any severe traffic noise effect (Fig. 5). 
This study shows that noise annoyance from different noise sources and stress-related symptoms (including tiredness, headache and difficulty concentrating) were common among occupants. Stress-related symptoms were associated with all types of noise annoyance. Exposure to many noise sources (a higher noise score) was related to more symptoms.

We found that personal factors, climate, degree of urbanization as well as building related factors can influence noise annoyance. Personal factors, such as younger age, female gender, living alone and a longer living time in the current residence were related to more noise annoyance. Females reported more noise from neighbours and were more sensitive to traffic noise causing severe effects. Occupants living in a warmer climate were more likely to be annoyed from noise at home, noise from amusement centre and noise from stairwell/elevators. Higher degree of urbanization (higher municipality population density) was related to more noise annoyance from neighbours, installations (lines and pipes), ventilation/fans/heat pumps, train traffic and flight traffic. Occupants living in rented dwelling were more annoyed from noise at home, noise from neighbours, amusement centre, installations (lines and pipes and stairwell/elevators), ventilation/fans/heat pumps, road traffic and train traffic. More noise annoyance were reported among those living in buildings constructed from $1961-1985$ Apartment situated above ground floor was related to more complaints of noise from neighbours and traffic noise causing severe effects. Having mechanical ventilation at home was related to more complaints of noise from ventilation/fans/warm pumps. Daily window opening was related to less noise annoyance from ventilation/fans/warm pumps but more noise annoyance from flight traffic.

\subsection{Prevalence of noise annoyance from building sources}

Reporting of any frequent noise at home was common in our study (11.2\%). We found only one previous study from Sweden investigating the prevalence of noise annoyance from different sources among residents. The study suggested that complaints of annoyance to traffic noise (8.7\%) and sound from neighbours (7.8\%) were most common among participants, followed by sounds from ventilation systems (3.9\%), sounds from other installations (2.4\%) and industry noise (1.1\%) (36).

Totally $13.2 \%$ reported voice/radio/TV/music/similar sounds from neighbours and $16.5 \%$ reported scraping sound/footsteps/thumping/similar sounds from neighbours. The prevalence found in our study is somewhat higher as compared to one previous Swedish study (36). Reporting of noise from amusement centre in the property was less common in our study (2.1\%).

A total of $8.9 \%$ reported noise from lines and pipes, and 10.5\% reported noise from stairwell/elevators. The prevalence of noise from installations in our study are higher as compared to one previous Swedish study, which showed a prevalence of $2.4 \%$ for noise from installation (36). Another study from Sweden suggested that the proportion of persons who were annoyed of noise from installations was more than twice as high as for traffic noise (25). The noises generated by installations, such as ventilation and air-conditioning systems, often have a dominant portion of low frequencies (20-200 $\mathrm{Hz})$ and may be a source of great annoyance and sleep disturbance. Thus, it can be important to regulate the noise exposure from sources such as installations.

We found that $7.6 \%$ were annoyed by noise ventilation/fans inside, and $4.5 \%$ were annoyed by noise from ventilation/fans/heat pumps from outside of the building. The prevalence found in our study is slightly higher than data from one previous Swedish study (36).

\subsection{Prevalence of noise annoyance from traffic}

We found that $16.1 \%$ reported noise from road traffic, $3.1 \%$ reported noise from train traffic and $3.3 \%$ reported noise from flight traffic. Road traffic and railway noise contribute significantly to the burden of disease in Sweden each year (use disability-adjusted life-years (DALY) measure) (37). The most important contributor to the disease burden (DALY) was sleep disturbances (54\%), followed by annoyance (30\%) and cardiovascular diseases (16\%) (37). Aircraft noise can be the most annoying noise source among all transportation noise sources when standardized for noise exposure level (5, 38, 39).

\subsection{Home environment factors in association with severe traffic noise effects}

The factor analysis for severe traffic noise effects identified two factors: the first factor included difficult to hear radio/TV, telephone calls being affected and normal conversations being affected; the second factor included rest/relaxation being affected, difficulties in sleeping and being woken up from traffic noise. We found that renting and living above ground floor were associated with severe traffic noise effects. Rented apartment buildings in Sweden were mostly built in 1960's and 1970's with poor internal sound insulation (thinner walls and only double glass windows). Those buildings are more likely to be located in poor areas such as major roads or train tracks. There can be noise remedies such as concrete walls to be placed between buildings and major roads/train tracks to reduce traffic noise. This kind of noise remedies can be effective to reduce noise levels for people living at ground floor or basement but less or not effective for people living above ground floor. Living at basement can be less disturbed by noise as small windows are often used in basement. Moreover, the ground connected to basement can help to reduce noise.

Noise is an environmental stressor that causes annoyance and interferes with daily activities (1). One Swedish study suggested that having access to quiet indoor and outdoor sections in one's dwelling was related to less activity interference among residents (40). Studies from Sweden and Brazil have investigated traffic noise in association with daily activity disturbances. Indoor activity disturbances (conversation, radio/TV, concentration, rest/relaxation, difficulties in falling asleep, awakenings, does not keep window open as often as desired) due to road traffic noise were much higher among those living in areas close to a main road than those living in quite areas in Sweden (41). Complaints of activity disturbances decreased in both areas after a two year period due to reduced traffic volume in both areas (41). Living in areas exposed to traffic noise, being aware of traffic noise in the home and considering the place of residence a noisy environment were predictors of noise annoyance among residents in Brazil (42). Living in areas exposing to traffic noise was associated more annoyance during activities, including watching TV, resting, talking and concentration (42).

\subsection{Noise annoyance from indoor and outdoor sources in association with stress-related symptoms}

Page $14 / 21$ 
Tiredness was the most commonly reported symptom in our study (23.1\%), followed by headache (8.5\%) and difficulty concentrating (5.5\%). We found that noise annoyance from noise at home, from sources outside of the building (road traffic, train traffic, flight traffic and ventilation), from sources inside of the building as well as reported severe traffic noise effects were all associated with stress-related symptoms (tiredness, headache and difficulty concentrating). Moreover, a higher noise score was related to increased risk of stress-related symptoms.

People annoyed by noise may experience a variety of negative responses, such as anger, disappointment, dissatisfaction, withdrawal, helplessness, depression, anxiety, distraction, agitation, or exhaustion $(10,43)$. The symptoms included in our study can be considered as stress-related psychosocial symptoms. Few studies have shown that noise annoyance and its relation with headache/tiredness. One experiment study from Sweden found that noise annoyance among university students in laboratory environment was positively associated with self-reported headaches (44). Another study from Sweden found that annoyance from road traffic noise among residents was related to tiredness (41). Moreover, one Swedish study suggested that exposing to sound levels from road traffic ranging from 45-68 dBA at the most exposed side may induce tiredness among residents (40). Some studies indicated that traffic noise exposure or traffic noise induced annoyance were associated with mental stress or mental disorders among residents (40, 41, 45, 46). The results in our study is in agreement with previous studies on noise annoyance from outdoor sources (mainly outdoor traffic) and stress symptoms/metal disorders. Our study indicate that noise annoyance from indoor sources (neighbours, installations and ventilation/fans/heat pumps) can affect stress-related symptoms, which is a new finding.

\subsection{Personal factors}

\subsubsection{Age}

Younger participants reported more noise annoyance as compared to older participants ( $>65 \mathrm{y}$ ), but the interaction analyses found that older participants were more sensitive to noise than younger participants. Moreover, we found that stress-related symptoms were more prevalent among younger participants as compared to older participants (> $65 \mathrm{y}$ ). Few studies have investigated age in association with noise annoyance. One study from India found that middle-aged subjects were more annoyed by road traffic noise as compared to younger and older subjects (47). One study from France indicated that older participants were more likely to be highly annoyed by aircraft noise (11). Younger participants in our study can have higher demands on their home environment. Another explanation can be that the effect of noise-induced annoyance in older people is concealed by physical consequences of age, with a strong increase of illnesses such as hearing loss.

\subsubsection{Gender}

We found that males reported less voice/radio/TV/music/similar sounds as compared to females in the mutual adjustment model (OR $95 \% \mathrm{Cl}, 0.75(0.61,0.91)$, $p=0.004)$. No gender difference was found regarding other noise complaints. According the interaction analyses, females were more sensitive to any severe traffic noise effect than males. We found no previous studies investigating gender in relation to noise annoyance from indoor sources.

\subsubsection{Living alone and living period in the current apartment}

We found that living alone was related more complaints of noise at home. The reason can be that persons living alone are not adopted to hearing other persons in the family, so they can be more sensitive to noise. A longer living time in the current apartment (more than 5 years) was associated with more reports of noise from neighbours. People with a longer living period in the current home can have less tolerance to noise and can be more sensitive to noise from neighbourhood.

\subsection{Climate zone}

Living in a warmer climate was related to more noise annoyance from any frequent noise at home, noise from amusement centre in the property and noise from stairwell/elevators inside of the building in our study. We found no previous studies on climate and noise annoyance. The reason is unclear and needs to be investigated in future studies. Warm climate can indicate more outdoor activities, especially in warmer seasons. People living in warmer climate are more used to open windows, spending time on balconies/outside the building in warmer seasons. Amusement centre can have open windows and have more people staying outside of the centre in warmer seasons. We are unclear about why living in a warmer zone is related to noise annoyance from stairwell/elevators.

\subsection{Urbanization}

We found that increased municipality population density was associated with more noise annoyance from neighbours, installations (lines and pipes), ventilation/fans/heat pumps, train traffic and flight traffic. A higher municipality population density indicates a higher degree of urbanization. A higher degree of urbanization often associates with increased exposure to environmental noise, especially from more frequent human activities in neighbourhood, from installations/ventilation systems in buildings nearby and from increased traffic intesity. Therefore, our results implies that measures for reducing environmental noise exposure in high populated large cities are needed, even in country like Sweden with a low population density.

\subsection{Building related factors}

\subsubsection{Ownership}

Renting was associated with noise annoyance from different sources in our study, including any frequent noise at home, noise from neighbours, amusement centre, installations (lines and pipes, stairwell/elevators), ventilation/fans/heat pumps, road traffic and train traffic. Previous Swedish study indicated that a large amount of multi-family buildings are owned by the community and used for renting (48). Multi-family buildings constructed from 1961-1975 in Sweden are mostly rented and prioritized for renovation in nowadays. Those buildings often have poor sound insulation due to thinner walls, no triple glass windows and poorer insulation in floors. Moreover, they can be located in areas with more traffic noise because such areas have lower land price. Families with lower 
socio-economic status are more likely to live in those buildings. Moreover, people living in rented building areas are more likely to have less social control as compared to those living in self-owned building areas. We found that participants living in self-owned apartments were more sensitive to any frequent noise at home and any severe traffic noise effect. This indicates that the associations between renting and noise annoyance indicators in our study cannot be explained by high noise sensitivity among participants living in rented apartments. It is more likely that participants living in rented apartments were exposed to more noise than those living in self-owned apartments (worse sound insulation, more noise from outside, worse social control/less respect between tenants).

\subsubsection{Building construction year}

We found that construction year from 1961-1985 was more likely to be associated with noise annoyance in general. We have found previously that subjects living in multi-family buildings built during 1961-1985 in Sweden had more respiratory illnesses (35). Multi-family buildings from 1961-1975 were constructed during a building boom in large cities in Sweden influenced by energy saving demands. New construction techniques and building materials were applied to reduce energy consumption during this period (48). It was reported that the prevalence of dampness problems was highest among those buildings (49). Those buildings were mostly poor quality high-rise buildings rented by poor people. They were constructed with thinner walls, poorer insulation in floors and only double glass windows (no triple glass windows). In Sweden, apartment buildings from 1976-1985 often have thinner walls with less sound insula and double glass windows. Newer apartment buildings have better insulation and tripple glazing. Thus, poor sound insulation can be the main reason to noise annoyance found in our study.

\subsubsection{Other building related factors}

Having mechanical ventilation was associated more complaints of noise from ventilation/fans/warm pumps. Noise from mechanical ventilation system can disturbs, especially in buildings with poor sound insulation. Reducing noise impact on residents when installing a mechanical ventilation system can be important.

Having a bathroom fan at home was related to noise annoyance from amusement centre. We have no explanation to this association which could be a chance finding.

Daily window opening was related to less complaints of noise from ventilation/fans/warm pumps. People with the habit of frequently opening windows can be less sensitive to constant noise from ventilation systems. In our study, daily window opening was associated with more complaints of noise from flight traffic. The reason is unknown. One study reported that residents who kept living room windows open were shown to be less annoyed by aircraft noise (50), which is opposite to our finding.

\subsection{Strengths and limitations}

\subsubsection{Strengths}

The present study is one of a few studies on noise annoyance that covered a sample of a whole country. All subjects ( $\geq 18$ years) living in multi-family buildings were included in the present study, with no prior information on their health status. There was no major difference in participation rate between different municipalities, between those being born in Sweden and foreign-born persons, and between Swedish citizens or non-Swedish citizens. Most of the differences in participation rate were small (35). Some factors discussed such as temperature zone and population density were not obtained from the selfadministered questionnaire, which reduces potential reporting bias.

\subsubsection{Limitations}

The response rate was not very high in our study (46\%). The participation rate was higher among elderly and married persons, but slightly lower in larger cities, suburban municipalities and in older buildings (35). Information bias can occur in questionnaire surveys. Subjects may overestimate or underestimate environment risk factors as well as stress-related symptoms. We found some associations between specific home environment risk factors and noise annoyance, not a similar association for all risk factors. Thus, our findings are less likely to be seriously biased by selection or information bias.

\section{Conclusions}

As shown in the present study, noise annoyance from different indoor and outdoor sources were common among occupants living in multi-family buildings in Sweden. Noise annoyance was related to tiredness, headache and difficulty concentrating. Exposure to noise from many sources was related to a higher risk of symptom.

Younger participants reported more noise annoyance and more stress-related symptoms as compared to older participants. Females can be more sensitive to noise from neighbours and traffic noise causing severe effects. Occupants living alone complained more often noise at home. Occupants having a longer living time in the current residence reported more often noise from neighbours. Living in a warmer climate was related to more noise annoyance from noise at home, noise from amusement centre and noise from stairwell/elevators. Higher municipality population density was associated with more noise annoyance from neighbours, installations (lines and pipes), ventilation/fans/heat pumps, train traffic and flight traffic. Renting was associated with noise annoyance from noise at home, noise from neighbours, amusement centre, installations (lines and pipes and stairwell/elevators), ventilation/fans/heat pumps, road traffic and train traffic. Occupants living in buildings constructed from 1961-1985 reported in general more noise annoyance. Living above ground floor was related to more complaints of noise from neighbours and traffic noise causing severe effects. Having mechanical ventilation was associated more noise annoyance from ventilation/fans/warm pumps. Daily window opening was associated with less complaints of noise from ventilation/fans/warm pumps but more complaints of noise from flight traffic. Our study suggested that personal factors (younger age, female gender, living alone, a longer living period in 
current apartment), a warmer climate zone, higher population density as well certain building factors (including renting, construction year from 1961-1985, living above ground floor, mechanical ventilation systems) were associated with more noise annoyance among occupants.

\section{Abbreviations}

\section{BETSI}

Building Energy, Technical Status and Indoor Environment

$\mathrm{Cl}$

confidence interval

DALY

disability-adjusted life-years

dBA

A-weighted decibels

OR

odds ratios

SCB

Statistics Sweden (In Swedish:Statistiska centralbyrån)

WHO

World Health Organization

\section{Declarations}

\section{Ethics approval and consent to participate}

The study was performed in accordance with the Declaration of Helsinki. The study and the consent procedure were approved by the Regional Ethical Committee in Uppsala, Sweden (reference number 2007-281). Informed consent was obtained from all participants. An information letter sent together with the questionnaire stated that if the subjects answered and returned the questionnaire it meant they had given informed consent.

\section{Consent for publication}

Not applicable.

\section{Availability of data and materials}

The datasets used and/or analyzed during the current study are available from the corresponding author on reasonable request.

\section{Competing interests}

The authors declare that they have no competing interests.

\section{Funding}

The study was funded by the Swedish AFA Insurance (No. 467801100). The funder had no role in the design of the study and collection, analysis, and interpretation of data and in writing the manuscript.

\section{Authors' contributions}

JW: Conceptualization; Data curation; Formal analysis; Funding acquisition; Investigation; Software; Validation; Visualization; Roles/Writing - original draft; Writing - review \& editing.

DN: Conceptualization; Data curation; Investigation; Methodology; Software; Supervision; Validation; Writing - review \& editing

All authors read and approved the final manuscript.

\section{Acknowledgments}

Thanks to Boverket and the Swedish National Board of Housing, Building and Planning. The authors would also like to thank all the participants.

\section{References}

1. World Health Organization. Burden of Disease from Environmental Noise. Quantification of Healthy Life Years Lost in Europe; WHO: Bonn, Germany, 2011.

2. Gupta A, Gupta A, Jain K, Gupta S. Noise Pollution and Impact on Children Health. Indian journal of pediatrics. 2018;85(4):300-6.

3. Seidman MD, Standring RT. Noise and quality of life. International journal of environmental research and public health. 2010;7(10):3730-8.

4. Daiber A, Kroller-Schon S, Frenis K, Oelze M, Kalinovic S, Vujacic-Mirski K, et al. Environmental noise induces the release of stress hormones and inflammatory signaling molecules leading to oxidative stress and vascular dysfunction-Signatures of the internal exposome. BioFactors (Oxford 
England). 2019.

5. World Health Organization (WHO). Guidelines for Community Noise; World Health Organization Europe: Geneva, Switzerland, 1999.

6. Noise in Europe 2014. European Environment Agency, 2014. No 10/2014. doi:10.2800/763331.

7. Bluhm G, Eriksson C. Cardiovascular effects of environmental noise: research in Sweden. Noise \& health. 2011;13(52):212-6.

8. Guski R, Schreckenberg D, Schuemer R. WHO Environmental Noise Guidelines for the European Region: A Systematic Review on Environmental Noise and Annoyance. International journal of environmental research and public health. 2017;14(12).

9. International Organization for Standardization, ISO 1996-1 Acoustics - Description, measurement and assessment of environmental noise - Part 1: Basic quantities and assessment procedures. Geneva: International Organization for Standardization; 2003.

10. Fields JM, De Jong RG, Gjestland T, Flindell IH, Job RFS, Kurra S, et al. STANDARDIZED GENERAL-PURPOSE NOISE REACTION QUESTIONS FOR COMMUNITY NOISE SURVEYS: RESEARCH AND A RECOMMENDATION. Journal of sound and vibration. 2001;242(4):641-79.

11. Lefèvre $M$, Chaumond $A$, Champelovier $P$, Giorgis Allemand $L$, Lambert $J$, Laumon $B$, et al. Understanding the relationship between air traffic noise exposure and annoyance in populations living near airports in France. Environment international. 2020;144:106058.

12. Bartels S, Márki F, Müller U. The influence of acoustical and non-acoustical factors on short-term annoyance due to aircraft noise in the field - The COSMA study. The Science of the total environment. 2015;538:834-43.

13. Guski R. Personal and social variables as co-determinants of noise annoyance. Noise \& health. 1999;1(3):45-56.

14. Fields JM. Effect of personal and situational variables on noise annoyance in residential areas. The Journal of the Acoustical Society of America. 1993;93(5):2753-63.

15. Hoeger R. Aircraft noise and times of day: possibilities of redistributing and influencing noise exposure. Noise \& health. 2004;6(22):55-8.

16. Klæboe R. Noise and Health: Annoyance and Interference. Elsevier B.V; 2011. p. 152-63.

17. Jakovljevic B, Paunovic K, Belojevic G. Road-traffic noise and factors influencing noise annoyance in an urban population. Environment international. 2009;35(3):552-6.

18. Kempen EV, Casas M, Pershagen G, Foraster M. WHO Environmental Noise Guidelines for the European Region: A Systematic Review on Environmental Noise and Cardiovascular and Metabolic Effects: A Summary. International journal of environmental research and public health. 2018;15(2).

19. Dzhambov AM, Dimitrova DD. Residential road traffic noise as a risk factor for hypertension in adults: Systematic review and meta-analysis of analytic studies published in the period 2011-2017. Environmental pollution (Barking, Essex: 1987). 2018;240:306 - 18.

20. Ndrepepa A, Twardella D. Relationship between noise annoyance from road traffic noise and cardiovascular diseases: a meta-analysis. Noise \& health. 2011;13(52):251-9.

21. Huang D, Song X, Cui Q, Tian J, Wang Q, Yang K. Is there an association between aircraft noise exposure and the incidence of hypertension? A metaanalysis of 16784 participants. Noise \& health. 2015;17(75):93-7.

22. Basner M, McGuire S. WHO Environmental Noise Guidelines for the European Region: A Systematic Review on Environmental Noise and Effects on Sleep. International journal of environmental research and public health. 2018;15(3).

23. Perron S, Tetreault LF, King N, Plante C, Smargiassi A. Review of the effect of aircraft noise on sleep disturbance in adults. Noise \& health. 2012;14(57):58-67.

24. Niemann H, Bonnefoy X, Braubach M, Hecht K, Maschke C, Rodrigues C, et al. Noise-induced annoyance and morbidity results from the pan-European LARES study. Noise \& health. 2006;8(31):63-79.

25. Persson Waye K, Bengtsson J, Agge A, Bjorkman M. A descriptive cross-sectional study of annoyance from low frequency noise installations in an urban environment. Noise \& health. 2003;5(20):35-46.

26. Boverket. Statistiska urval och metoder i Bovekets projekt BETSI. Sweden: The Swedish National Board of Housing, Building and Planning; 2010. Report No.: 978-91-86342-48-7\$4

27. Norback D, Bjornsson E, Janson C, Palmgren U, Boman G. Current asthma and biochemical signs of inflammation in relation to building dampness in dwellings. The international journal of tuberculosis and lung disease: the official journal of the International Union against Tuberculosis and Lung Disease. 1999;3(5):368-76.

28. Bjerg A, Ekerljung L, Middelveld R, Dahlen SE, Forsberg B, Franklin K, et al. Increased prevalence of symptoms of rhinitis but not of asthma between 1990 and 2008 in Swedish adults: comparisons of the ECRHS and GA(2)LEN surveys. PloS one. 2011;6(2):e16082.

29. Burney PG, Luczynska C, Chinn S, Jarvis D. The European Community Respiratory Health Survey. The European respiratory journal. 1994;7(5):954-60.

30. Janson C, Anto J, Burney P, Chinn S, de Marco R, Heinrich J, et al. The European Community Respiratory Health Survey: what are the main results so far? European Community Respiratory Health Survey II. The European respiratory journal. 2001;18(3):598-611.

31. Andersson K. Epidemiological approach to indoor air problems. Indoor air. 1998;8(S4):32-9.

32. Engvall K, Norrby C, Norback D. Ocular, nasal, dermal and respiratory symptoms in relation to heating, ventilation, energy conservation, and reconstruction of older multi-family houses. Indoor air. 2003;13(3):206-11.

33. Lista över Sveriges kommuner [Available from: http://sv.wikipedia.org/wiki/Lista_\%C3\%B6ver_Sveriges_kommuner. Accessed 1 July 2021.

34. The Swedish National Board of Housing, Building and Planning. Enkätundersökning om boendes upplevda inomhusmiljö och ohälsa - resultat från projektet BETSI. Sweden: The Swedish National Board of Housing, Building and Planning; 2009.

35. Wang J, Engvall K, Smedje G, Norback D. Rhinitis, asthma and respiratory infections among adults in relation to the home environment in multi-family buildings in Sweden. PloS one. 2014;9(8):e105125.

Page $18 / 21$ 
36. Persson R, Björk J, Ardö J, Albin M, Jakobsson K. Trait anxiety and modeled exposure as determinants of self-reported annoyance to sound, air pollution and other environmental factors in the home. International archives of occupational and environmental health. 2007;81(2):179-91.

37. Eriksson C, Bodin T, Selander J. Burden of disease from road traffic and railway noise - a quantification of healthy life years lost in Sweden. Scand J Work Environ Health. 2017;43(6):519-25.

38. Miedema HM, Oudshoorn CG. Annoyance from transportation noise: relationships with exposure metrics DNL and DENL and their confidence intervals. Environmental health perspectives. 2001;109(4):409-16.

39. Brink M, Schäffer B, Vienneau D, Foraster M, Pieren R, Eze IC, et al. A survey on exposure-response relationships for road, rail, and aircraft noise annoyance: Differences between continuous and intermittent noise. Environment international. 2019;125:277-90.

40. Öhrström E, Skånberg A, Svensson H, Gidlöf-Gunnarsson A, Institutionen för medicin afsof, Sahlgrenska a, et al. Effects of road traffic noise and the benefit of access to quietness. Journal of sound and vibration. 2006;295(1):40-59.

41. Ohrström E. Longitudinal surveys on effects of changes in road traffic noise-annoyance, activity disturbances, and psycho-social well-being. J Acoust Soc Am. 2004;115(2):719-29.

42. Paiva KM, Cardoso MRA, Zannin PHT. Exposure to road traffic noise: Annoyance, perception and associated factors among Brazil's adult population. The Science of the total environment. 2019;650(Pt 1):978-86.

43. Lekaviciute J, Argalasova-Sobotova L. Environmental noise and annoyance in adults: research in Central, Eastern and South-Eastern Europe and Newly Independent States. Noise \& health. 2013;15(62):42-54.

44. Ohrström E, Björkman M, Rylander R. Noise annoyance with regard to neurophysiological sensitivity, subjective noise sensitivity and personality variables. Psychol Med. 1988;18(3):605-13.

45. Koszarny Z. [The estimate of well-being and self-assessed health status in urban population in various acoustic areas]. Rocz Panstw Zakl Hig. 2001;52(2):165-78.

46. Baudin C, Lefèvre M, Champelovier P, Lambert J, Laumon B, Evrard AS. Aircraft Noise and Psychological III-Health: The Results of a Cross-Sectional Study in France. International journal of environmental research and public health. 2018;15(8).

47. Banerjee D. Road traffic noise exposure and annoyance: a cross-sectional study among adult Indian population. Noise \& health. 2013;15(66):342-6.

48. Engvall K, Norrby C, Bandel J, Hult M, Norback D. Development of a multiple regression model to identify multi-family residential buildings with a high prevalence of sick building syndrome (SBS). Indoor air. 2000;10(2):101-10.

49. Engvall K, Norrby C, Norback D. Sick building syndrome in relation to building dampness in multi-family residential buildings in Stockholm. International archives of occupational and environmental health. 2001;74(4):270-8.

50. Babisch W, Swart W, Houthuijs D, Selander J, Bluhm G, Pershagen G, et al. Exposure modifiers of the relationships of transportation noise with high blood pressure and noise annoyance. J Acoust Soc Am. 2012;132(6):3788-808.

\section{Figures}

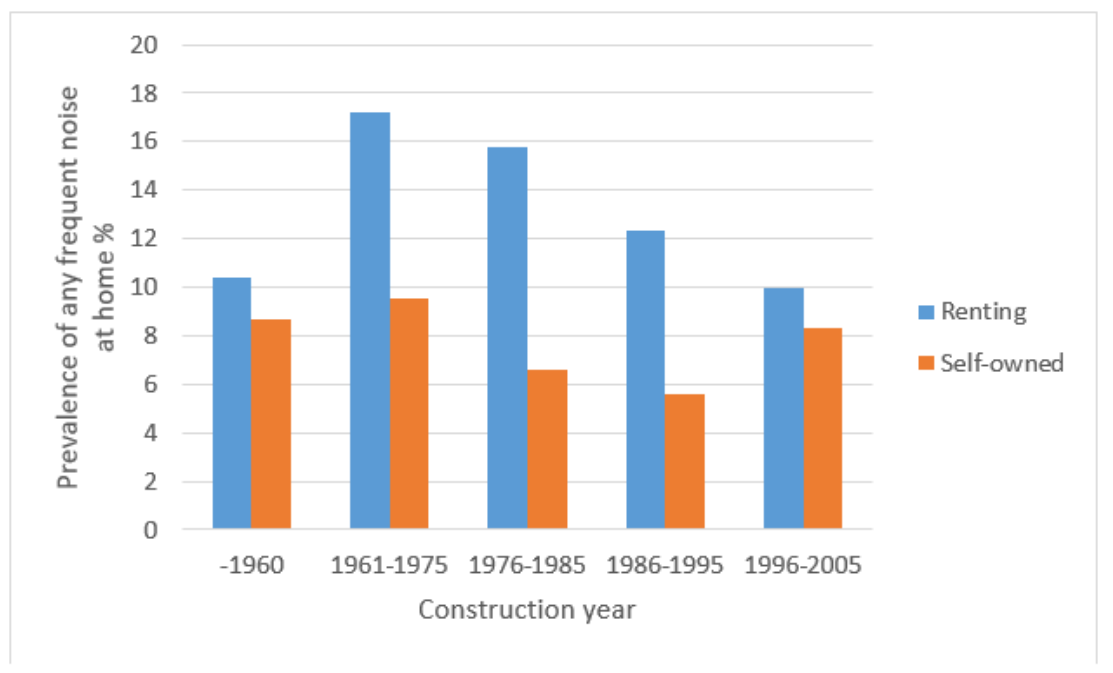

Figure 1

Prevalence of any frequent noise at home in different construction periods, stratified by ownership. 


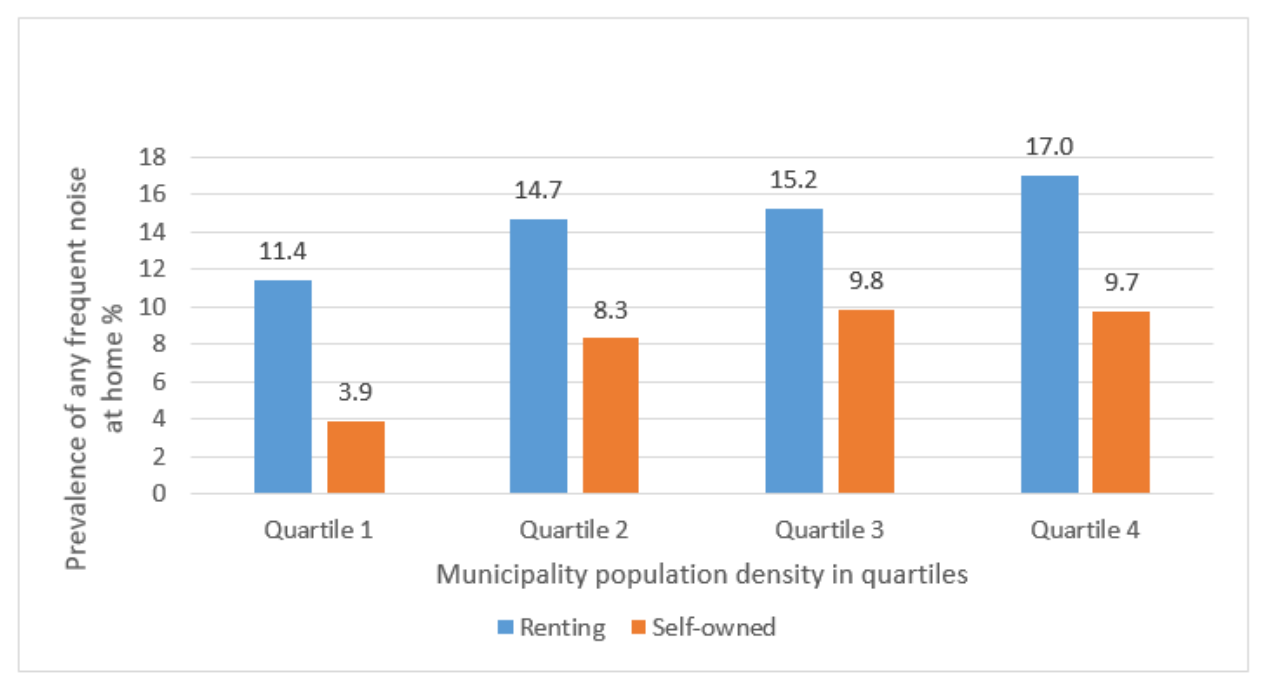

\section{Figure 2}

Prevalence of any frequent noise at home in relation to urbanization, stratified by ownership.

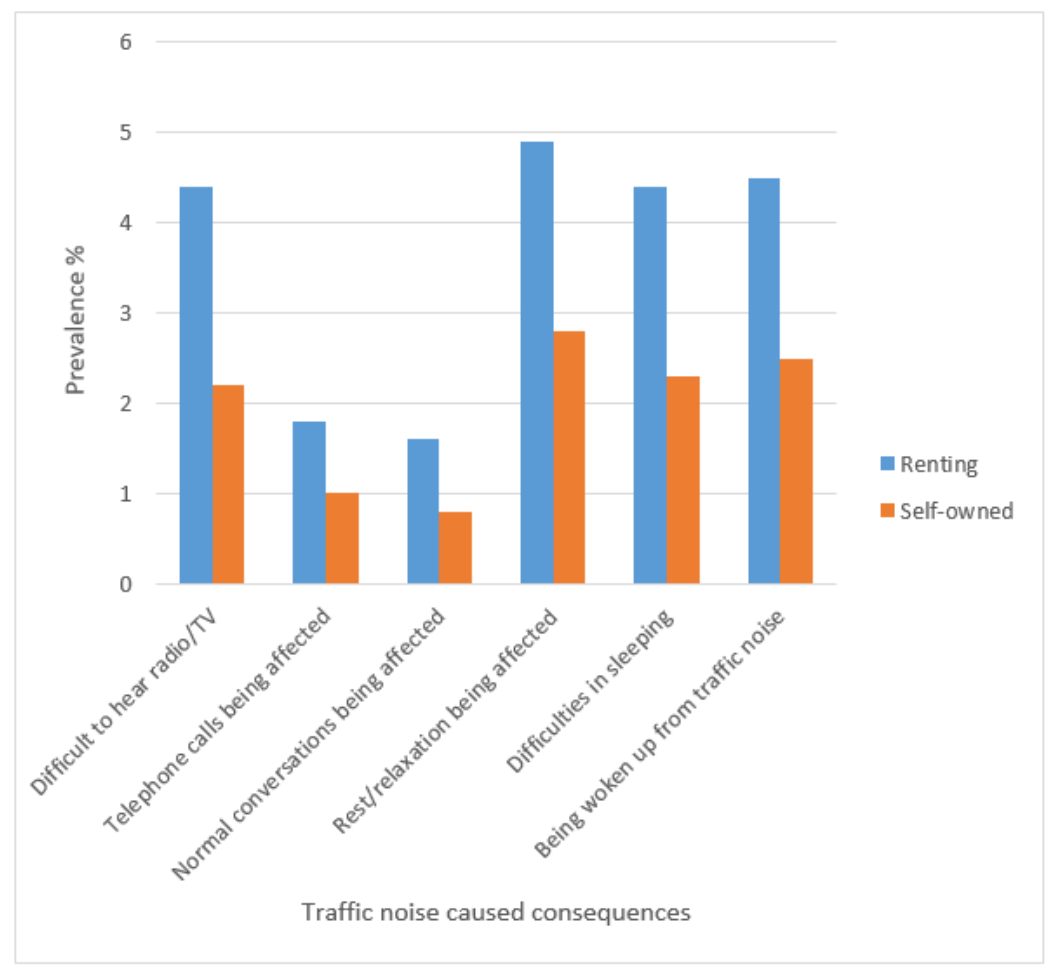

\section{Figure 3}

Prevalence of different severe traffic noise effects, stratified by ownership. 


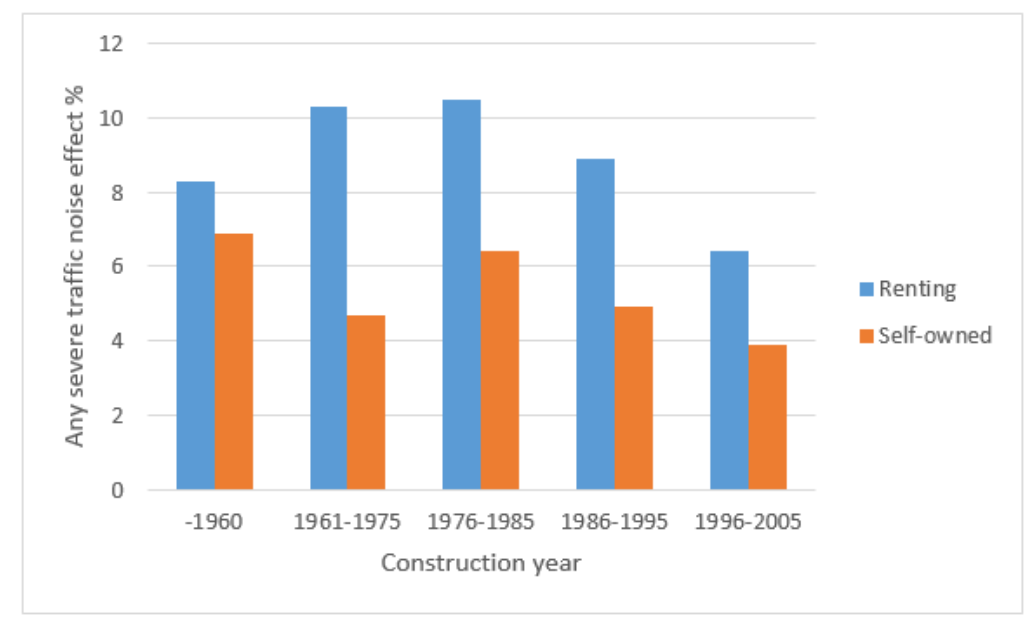

\section{Figure 4}

Prevalence of any severe traffic noise effect in different construction periods, stratified by ownership.

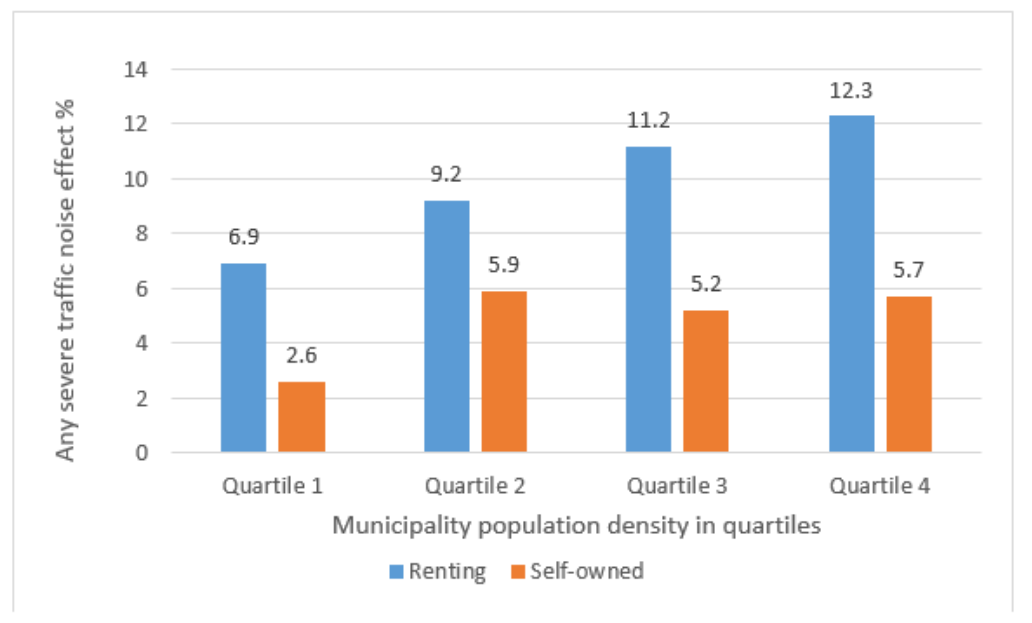

Figure 5

Prevalence of any severe traffic noise effect in different population quartiles, stratified by ownership.

\section{Supplementary Files}

This is a list of supplementary files associated with this preprint. Click to download.

- Additionalfile1BETSI20210712.docx 